\title{
Understanding Thermal Conductance across Multi-Wall Carbon Nano- tube Contacts: Role of Nanotube Curvature
}

Vikas Varshney, ${ }^{1,2, *}$ Jonghoon Lee ${ }^{1,2,}$ Deyu Li, ${ }^{3}$ Joshua S. Brown, ${ }^{4, \$}$ Barry L. Farmer, ${ }^{1}$ Andrey A. Voevodin, ${ }^{1,8}$ and Ajit K. Roy ${ }^{1, *}$

\begin{abstract}
Thermal energy transfer at the interconnects in carbon based nanoelectronic devices plays a crucial role towards their performance as well as their reliability. In this study, we investigate such thermal energy transfer across physically interacting multi-wall carbon nanotubes (MWCNTs) as a function of their diameter, length, number of walls, inter-layer chirality differences, and different angular orientation of the cross-contact. Using non-equilibrium molecular dynamics simulations for phonon energy transfer, we predict that MWCNTs' curvature and their number of walls emerge as two critical factors, with each of them determining the limiting value of the thermal conductance across MWCNT contacts in different diameter regimes. For thinner MWCNTs, the curvature determines the limiting value of the conductance and leads to an interesting nonmonotonic character, while the number of walls dominates the contact conductance for large diameter MWCNTs. We discuss their respective origins and distinguish their governing regimes using several arguments -focusing of phonons, and confinement of the phonon focusing cone, large mean free path of graphite- and how they modulate radial thermal transport, leading to observed trends of thermal conductance across MWCNT contacts.
\end{abstract}

KEYWORDS: Molecular Dynamics, MWCNTs, Thermal Transport, Interface Thermal Conductance, Phonon Focusing

Present Addresses:

\$Electrical Energy and Computer Engineering Department, University of Colorado, 1111 Engineering Dr., Boulder, CO 80309-0422

${ }^{\S}$ Materials Science and Engineering, University of North Texas, Denton, TX 76203-5017 USA 


\section{Introduction}

Interfaces play a pertinent role in thermal transport related structure-property relationships in many different areas of scientific interests, including composites and electronics reliability. As the system dimensions reduce to sub-micron/nanometer length scales, their role becomes even more critical due to increase in surface-area to volume ratio. Within the specialized field of nanoelectronics, fabrication of carbon nanotube (CNT) based devices have attained consistent attention [1], partly due to their excellent electrical and thermal transport properties, which can be further tuned through chemical and structural modifications [2-5]. For example, metallic CNTs (especially multi-wall carbon nanotubes (MWCNTs)) have been used as percolation constituents in flexible traces, conductive solders and nanocomposites for desired electronic transport [6-13]. However, despite a large number of potential nanoelectronic applications, the use of CNT devices has still been limited because of their unknown reliability and variation in performance, thus advocating the necessity of understanding the energy transfer and loss at device interconnects. As electrons pass through and hop across different CNTs within these device components, inevitable heating due to dissipative losses at the interconnects (and/or the physical contacts) emerges as a performance-limiting bottleneck. Of particular interest to this study is the issue of thermal conductance across CNT contacts, which are often experimentally encountered in nanotube based flexible solders, adhesives and polymeric nanocomposite materials tailored for electronics applications [6-14].

To date, most experimental work towards measuring interface thermal conductance (ITC) across CNT contacts has been done on ensembles of CNTs, where ITC is indirectly estimated as an average over numerous contacts [15-18]. Recently, Yang et al. reported an interesting observation through one of the first direct measurements of ITC between individual MWCNTs of 42-68 $\mathrm{nm}$ diameter [19]. The experimental data indicated that the area-normalized thermal conductance between two MWCNT segments (broken from one long MWCNT) increases approximately linearly with tube diameter. Interestingly, even for the largest MWCNTs, the reported value was still an order of magnitude lower than that of inter-layer thermal conductance in bulk graphite $\left(\sim 18 \mathrm{GWm}^{-2} \mathrm{~K}^{-1}\right)$ [3]. This behavior is surprising with common expectation that the normalized ITC values should be independent of tube diameter. Through semi-quantitative analyses, the authors attributed their observation to three coupled mechanisms: phonon reflection at 
inner tube surface, long phonon mean free path along $c$-axis of graphite, and significant phonon focusing in graphitic materials because of its high thermal anisotropic nature. Despite their numerical analyses were based on simple slit contact model between two semi-infinite graphitic slabs, the study provided valuable physical and intuitive insights into the observed phenomenon [19].

The planar graphitic model, as employed by Yang et al. appears adequate to model thermal exchange between large diameter (>40 nm) MWCNTs, where local curvature effects are marginal. However, for thinner CNTs, where intrinsic curvature can become significant, it is pertinent to ask following questions: a) Does the flat-graphitic model of contact thermal conductance still valid for such MWCNTs where their intrinsic curvature starts playing a role?; b) If so or not, how does curvature modulate or alter the aforementioned mechanisms towards governing interface thermal transfer?; c) In addition to diameter, what role does the number of inner walls play in determining overall thermal energy exchange?; d) Do chirality differences between consecutive walls play any role in inter-wall thermal energy exchange?; and e) How does the differences in angular orientation at MWCNT contact affect thermal transport across the contact?

Currently, there exists a disconnection between experimental and simulation efforts from the perspective of addressing questions projected above. While most thermal conductance experiments have been performed on MWCNTs (diameter > $20 \mathrm{~nm}$ up to 100s of nm) [15-19] primarily due to experimental challenges, almost all numerical modeling has been performed on singlewall CNTs (SWCNTs) (diameter $<2 \mathrm{~nm}$ ), because of the limitations associated with computational costs [20-34]. In this study, we aim to bridge this gap by performing comprehensive molecular dynamics (MD) simulations with more realistic models for the first time, i.e., modeling contact thermal conductance between MWCNTs up to $8 \mathrm{~nm}$ diameter and up to 10 walls along with modeling their flat graphitic counterparts, i.e., multi-layer graphene nano-ribbons (MLGNRs). The obtained results disclose interesting phenomena beyond what have been reported so far. The new observations, together with the underlying mechanism provide a more complete physical picture of contact thermal conductance between graphitic nanomaterials and help determining the efficiency of energy transport across device interconnects/junctions and thus, dictating their performance. 

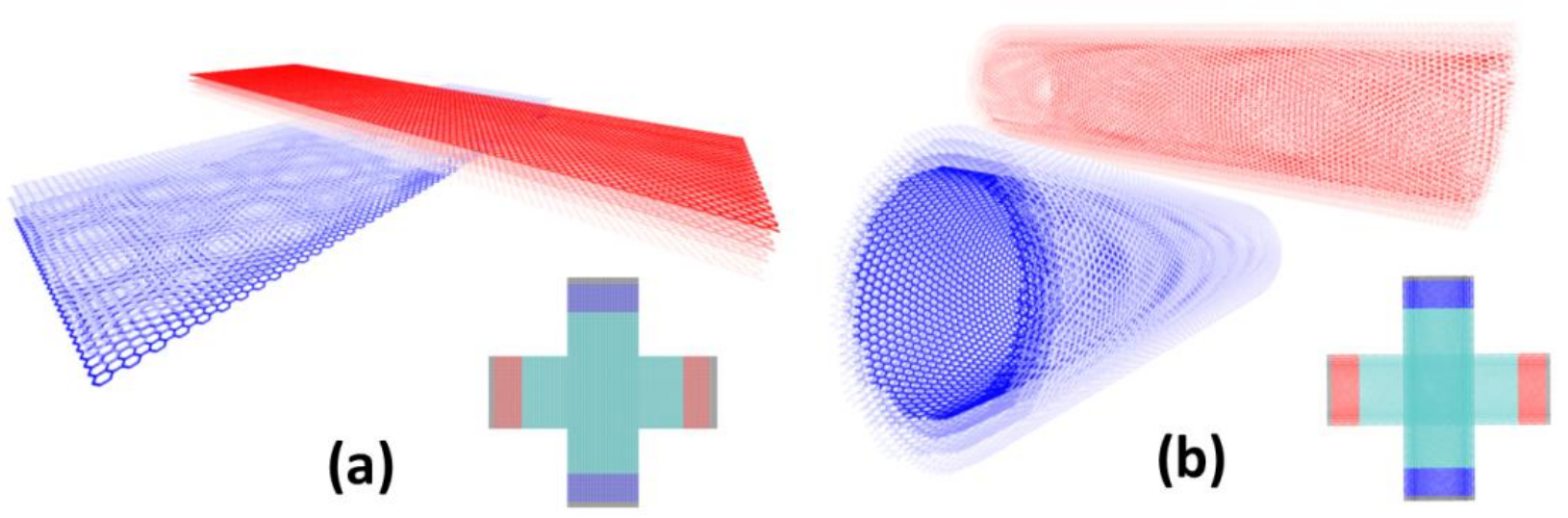

Figure 1: Perspective view of the schematic configurations of a representative (a) MLGNR and (b) MWCNT system in a cross-contact geometry as employed for interface thermal conductance simulations. The specific initial configurations are shown for (a) 4 layers of graphene nano-ribbons (ribbon width $7.8 \mathrm{~nm}$ ), and (b) $25 \mathrm{~nm}$ long, 4-wall MWCNT with outer CNT diameter $7.8 \mathrm{~nm}$. Different color shades for different walls (layers) in main figure are shown for visualization purposes. The insets in both schematics (visualized in orthographic orientation from top) represent the methodology used for thermal transport simulations as discussed in Methods section. Inset color schemes: fixed carbon atoms (black); hot thermostated carbon atoms (red); cold thermostated carbon atoms (blue); unthermostated carbon atoms (cyan).

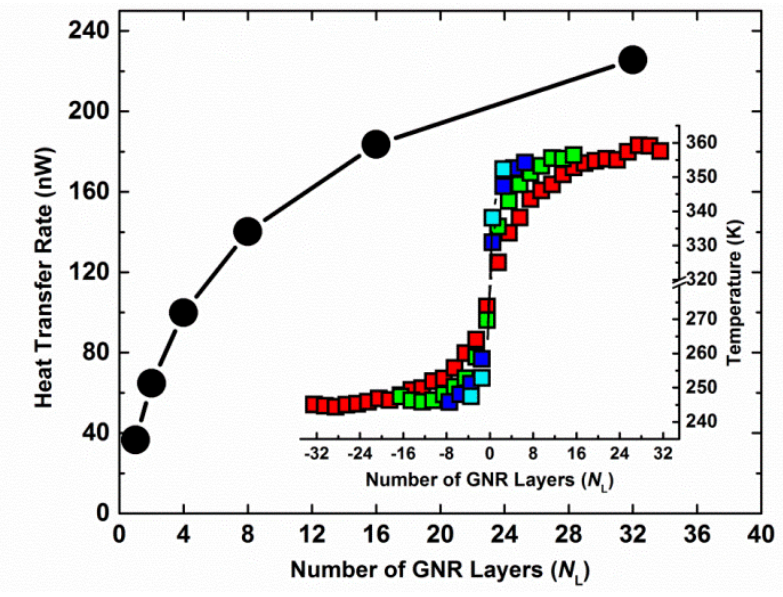

(a)

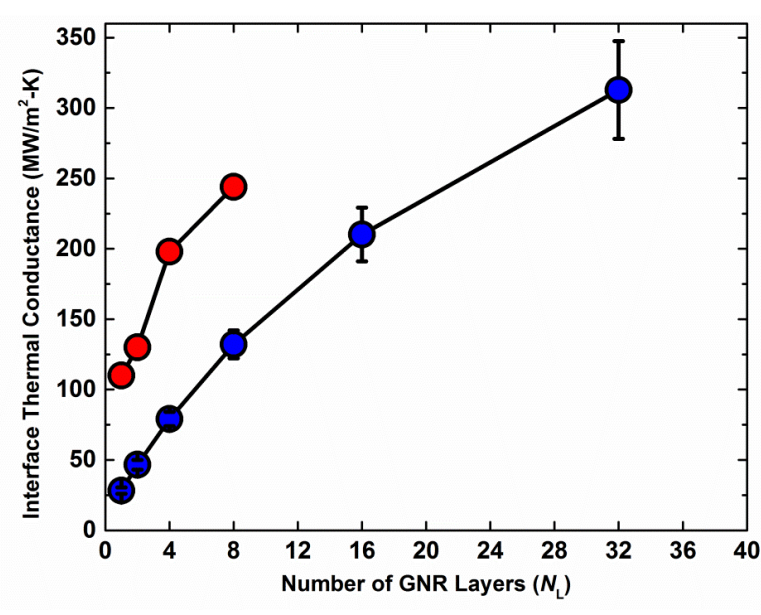

(b)

Figure 2: (a) Plot showing heat transfer rate (black circles) for different studied $25 \mathrm{~nm}$ long MLGNR systems. Steady-state temperature distribution across MLGNRs is shown in inset. Inset color scheme: 4 layers (cyan); 8 layers (blue); 16 layers (green); 32 layers (red). (b) Plot showing calculated interface thermal conductance across MLGNRs contacts for different number of studied multi-layers (blue circles). The simulation data from Yang et al. are presented in red circles. 


\section{Results and Discussion}

\subsection{Thermal Conductance across Multi-layer Graphene Nanoribbons}

We first verify the physical insights based on graphene stacks by performing non-equilibrium molecular dynamics (NEMD) simulations with MLGNRs. The modeled system is represented in Fig. 1a with two $\sim 8 \mathrm{~nm}$ wide, $\sim 25 \mathrm{~nm}$ long, 4-layer GNRs forming a cross contact. The simulation details and further specifics are discussed in the Methods section. We should point out that our simulations are notably different from what has been reported by Yang et al. in that (a) no periodic boundary conditions have been applied so this model truly represents two MLGNRs making a cross contact, unlike the previous study in which parallel, periodic GNR stacks were used; and (b) MLGNRs up to 32 layers (4 times to what is reported in Ref. [19]) were modeled, resulting in the effective thickness up to $\sim 11 \mathrm{~nm}$.

Fig. 2a shows the resultant heat transfer rate and the steady-state temperature distribution (in the inset) across MLGNRs as a function of the number of GNR layers $\left(N_{\mathrm{L}}\right)$, while Fig. 2b plots the derived contact thermal conductance as a function of $N_{\mathrm{L}}$. It is evident from Fig. 2 that both heat transfer rate and contact thermal conductance increase as $N_{\mathrm{L}}$ gets larger, which is consistent with the experimental observation based on MWCNTs and associated numerical analysis [19]. It should be noted that the maximum ribbon thickness $(\sim 11 \mathrm{~nm})$ is comparable to the wall thickness values of the MWCNTs used by Yang et al. ( 14-26 nm); and as indicated in Fig. 2b, the escalation of the contact thermal conductance with the ribbon thickness does not slow down significantly till 32 layers, suggesting that the observed trend is expected to persist for much thicker GNRs.

It is important to point out that without periodic boundary conditions, our simulation better represents two graphitic nanomaterials making a point contact, while the modeling results in Ref. 19 are actually for two flat plates making a slit contact in a periodic geometry. The consistency between these two approaches, i.e. observation of increasing trend in conductance with $N_{\mathrm{L}}$, is suggestive of marginal differences associated with applying different boundary conditions. The differences in absolute values of predicted interface thermal conductance (a factor of $\sim 2$ with respect to Ref. [19]) are attributed to differences in the modeling geometry, but more importantly to the differences in aspect ratio of modeled MLGNRs (lower aspect ratios were modeled in this 
study). It has been recently reported that GNR aspect ratio do play an important role towards outof-plane interface thermal conductance and lead to larger values at higher aspect ratios [35].

\subsection{Thermal Conductance across Multi-Wall Carbon Nanotubes}

After discussing and validating the cases where effects of tube curvature can be neglected and inter-tube thermal energy exchange in large MWCNTs can be simplified using their flat counterparts, i.e. flat graphene stacks, we switch our focus to regime of thinner MWCNTs $(<10 \mathrm{~nm})$, where curvature is not marginal and should not be neglected or simplified by modeling flat graphene-like cases. In this context, we perform a series of comprehensive MD simulations to calculate interface thermal conductance across MWCNT cross-contacts, where we vary the diameter of MWCNTs from 1.6 to $8 \mathrm{~nm}$, number of concentric walls ranging from 1 to 10 , and model MWCNT lengths of 25 and $50 \mathrm{~nm}$. A representative cross-contact geometry of interacting MWCNTs is shown in Fig. 1b. The specific details of simulation setup, their execution and thermal transport methodology are explained in Methods section.

One important issue to be discussed and appreciated is how to determine the contact area between interacting MWCNTs. Since the CNTs are intrinsically cylindrical and yet flexible, the 'effective' interaction area for thermal coupling at the contact between two tubes is not trivial to calculate and several methodologies have been employed in the literature to explore it $[21,23$, $25,28,36]$. The interaction is van der Waals in nature, which is of a very short range $(\sim 1 \mathrm{~nm})$,

and decays very quickly ( $\sim d^{-6}$ where $d$ is the distance between two atoms). In order to have a measure of the effective interaction area in the case of CNTs, we used a methodology similar to Evans et al. [21] In this scheme, the van der Waals interaction energy between two layers of flat graphene sheets is calculated for known area and then used to calculate the effective area of interaction $\left(A_{\mathrm{MW}}\right)$ as follows.

$$
A_{\mathrm{MW}}=\frac{E_{\mathrm{MW}}}{E_{\mathrm{G}}} A_{\mathrm{G}}
$$

where $E_{\mathrm{G}}$ and $A_{\mathrm{G}}$ are the interaction energy and the known area between bi-layer physically interacting graphene, respectively. $E_{\mathrm{MW}}$ is the van der Waals interaction energy between outermost CNTs (in MWCNTs). 


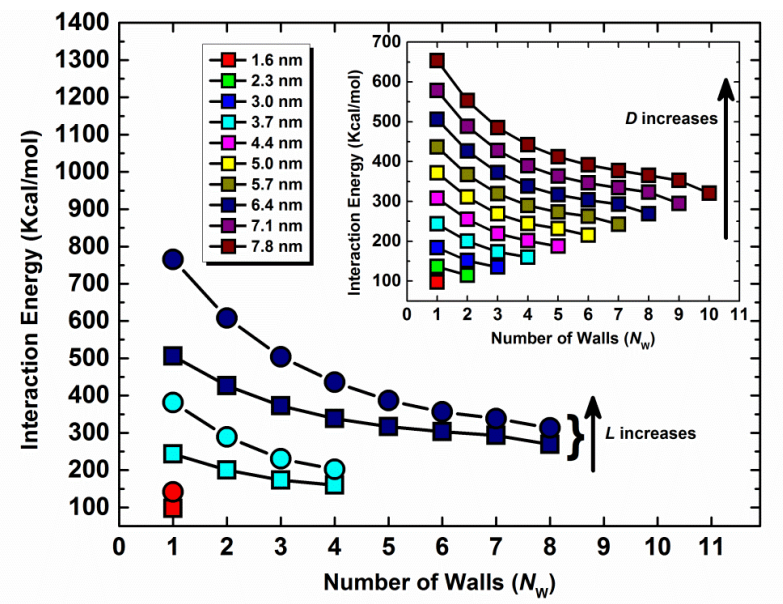

(a)

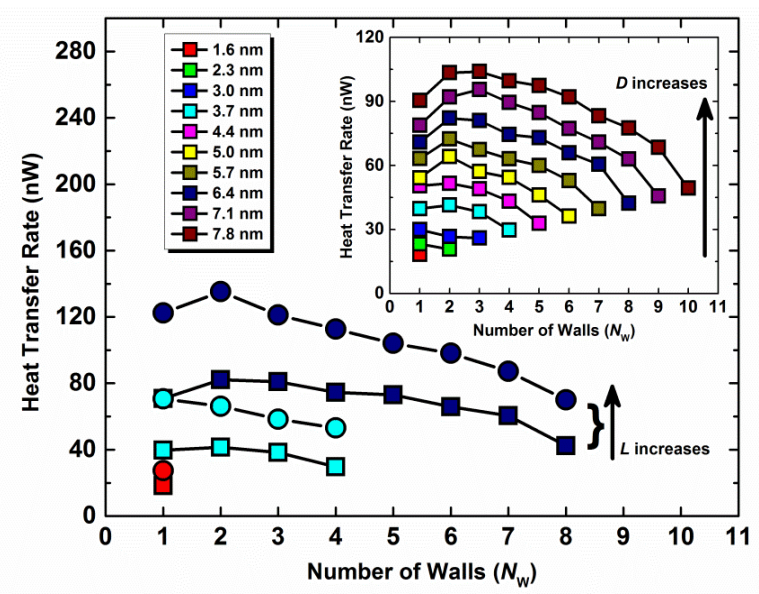

(b)

Figure 3: (a) Plots showing comparison and trends of interaction energy as a function of number of walls ( $\left.N_{\mathrm{W}}\right)$ for $25 \mathrm{~nm}$ (squares) and $50 \mathrm{~nm}$ (circles) long MWCNTs. Inset plots the interaction energy for all studied $25 \mathrm{~nm}$ long MWCNTs as a function of $N_{\mathrm{W}}$. (b) Plots showing comparison and trends of heat transfer rate as a function of number of walls for $25 \mathrm{~nm}$ (squares) and $50 \mathrm{~nm}$ (circles) long MWCNTs. Inset plots the heat transfer rate for all studied $25 \mathrm{~nm}$ long MWCNTs as a function of $N_{\mathrm{W}}$. In both figures, color scheme identifies the outermost CNT diameter.

Fig. 3a shows how the interaction energy $\left(E_{\mathrm{MW}}\right)$ between MWCNTs gets affected with respect to diameter of the outer-most wall $(D)$, number of walls $\left(N_{\mathrm{W}}\right)$, as well as their length $(L)$. Three trends are clearly evident from the figure. First, as seen from the inset in Fig. 3a, the interaction energy between MWCNTs increases monotonically with $D$ for constant $N_{\mathrm{W}}$ and $L$, which reflects the larger interaction area for larger diameter tubes. Secondly, for constant $L$ and $D, E_{\mathrm{MW}}$ decreases with increasing $N_{\mathrm{W}}$, which can be attributed to the reduction in contact zone area due to the added stiffness associated with increased intrinsic curvature of the MWCNTs (Please refer to Supporting information S3 for pictorial representation). Last but not least, for constant $D$ and $N_{\mathrm{W}}$, it is also observed that $E_{\mathrm{MW}}$ increases with MWCNT length. This observation is attributed to larger degree of local deformation near the contact region (resulting in increase in contact zone area) as longer MWCNTs are expected to be more flexible. Furthermore, it is also noted that this effect is greater for MWCNTs with less number of walls (small $N_{\mathrm{W}}$ ) since outermost CNT can locally deform to a much larger degree when the MWCNT (as a whole) is not intrinsically stiff.

Fig. 3b plots the total heat transfer rate, i.e., thermal energy exchange rate across crosscontact MWCNT interconnect, with respect to $D, L$ and $N_{\mathrm{W}}$. Resembling the interaction energy, the heat transfer rate also show two similar trends; (1) it increases with $D$ for constant $L$ and $N_{\mathrm{W}}$, and (2) it increases with $L$ for constant $D$ and $N_{\mathrm{W}}$. These trends fittingly correlate with the magnitude of $E_{\mathrm{MW}}$ (thus $A_{\mathrm{MW}}$ ) at the contact, i.e., the larger interaction energy leads to higher heat 
transfer rate. In addition, Fig. 3b indicates a much more interesting dependence of the total heat transfer rate on the number of walls. For small diameter CNTs $(<\sim 4 \mathrm{~nm})$, the heat transfer rate decreases monotonically with increasing $N_{\mathrm{w}}$ but for large diameter CNTs $(>\sim 4 \mathrm{~nm})$, the heat transfer rate shows a non-monotonic behavior, i.e., it first increases and then decreases as $N_{\mathrm{w}}$ gets larger.

The overall decreasing trend of total heat transfer rate with $N_{\mathrm{w}}$ is unexpected at first glance, given the monotonic increase of thermal conductance with $N_{\mathrm{w}}$ (i.e., graphene stacks in Fig. 2a) as reported in MWCNT experiments (and flat graphene stack simulations) [19] and thus, clearly showcases the importance of MWCNT curvature on thermal energy exchange. The observed non-monotonic behavior arises due to contributions from two competing effects, positive contributions from additional inner walls and negative contribution from decrease in interaction area due to stiffness, which will be discussed shortly in greater detail. Finally, it is also noted on the close observation that decreasing trends of $E_{\mathrm{MW}}$ and total heat transfer rate are not identical with respect to $N_{\mathrm{W}}$. At higher $N_{\mathrm{W}}$, while the decrease in interaction energy is gradual, the decay in heat transfer rate is more pronounced.

To better understand the complex interaction between the total heat transfer rate and the number of walls, we seek to normalize the heat transfer rate with the contact area, i.e., solve for the contact thermal conductance per unit area. Figs. $\mathbf{4 a}$ and $\mathbf{4 b}$ plot the normalized contact thermal conductance $(\Lambda)$ with respect to both NW and the CNT diameter for cases with $25 \mathrm{~nm}$ and $50 \mathrm{~nm}$ long MWCNTs, respectively. The area for $\Lambda$ prediction is calculated using Eq. 1. The temperature drop $(\Delta T)$ at the interface was calculated as the temperature difference between the outermost walls of the two MWCNTs near the contact after steady-state was reached (a representtative dataset for hot and cold CNT temperatures along with their standard error are provided in Supporting Information S6).

Both figures show a very intriguing non-monotonic behavior of thermal conductance with respect to number of walls $\left(N_{\mathrm{W}}\right)$, which has not been reported thus far. Furthermore, it is also observed that the thermal conductance is relatively insensitive to diameter for small $N_{\mathrm{W}}$, but increases (followed by eventual saturation) with diameter for large $N_{\mathrm{W}}$. These results and trends cannot be explained using well-established interface thermal conductance models, such as acous- 
tic and diffused mismatch models [37], as contact resistance in these models is determined by the materials in contact and the morphology of the interface, but is not related to the size of the system as shown in our results. Similarly, ballistic (Sharvin) resistance model [38] is also inadequate in present context as this resistance does not change with contact size, which is also different from our observed results. We believe that this inadequacy arises from anisotropic nature of thermal transport in multi-layer graphitic structures such as MWCNTs. We discuss our findings based on various phonon effects (phonon focusing and reflection as well as large mean free path) observed in thermally anisotropic materials $[19,39,40]$, and propose a few phenomenological arguments for how these effects are modified when tube curvature becomes important in MWCNTs. First, we describe a few premises for our arguments and use them to discuss the origin of non-monotonic behavior of contact conductance as a function of $N_{\mathrm{W}}$ for a constant diameter MWCNT. After that, we provide a short discussion on how this behavior is modified with respect to diameter, length, and interlayer chirality differences of MWCNTs. The following discussion is based on three premises;

a) the phonons travelling along radial direction in MWCNTs are focused along that direction due to highly anisotropic nature of thermal conductivity of MWCNTs with very different interaction potentials within each tube and across different tube layers. We term this as "phonon focusing effect" which is often observed in materials with high thermal anisotropy such as graphite. It has been suggested that the phonon transport along the radial direction of MWCNT is very similar to that along the cross-plane direction of graphite, for which the term "phonon focusing" is applicable $[15,39]$. Here, the normal direction of the contact, i.e, radial direction, can be treated as equivalent to the stack of carbon atoms in a graphite-like geometry along with a curvature. In addition, for cross-plane (radial) phonons with respect to the contact area between the two MWCNTs, the allowable propagation directions form a cone, which gets narrower with increase in CNT stiffness. This premise is schematically shown in Fig. 5;

b) like flat graphitic case, phonon reflection occur at free surfaces (inner-wall CNTs for our case); and

c) only the phonons that are emitted from hot MWCNT and thermalized in cold MWCNT through three-phonon scattering process contribute to thermal energy transfer, while phonons that are reflected back into the emitting tube without thermalization scattering do not. 


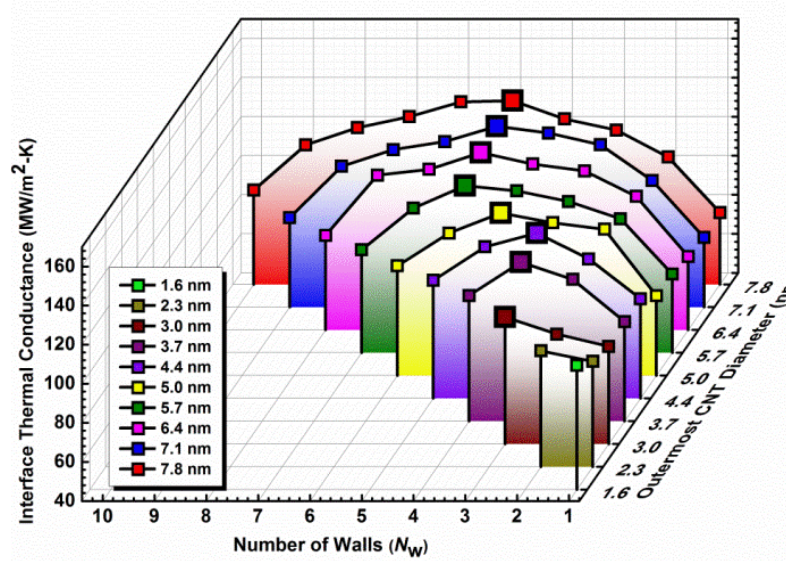

(a)

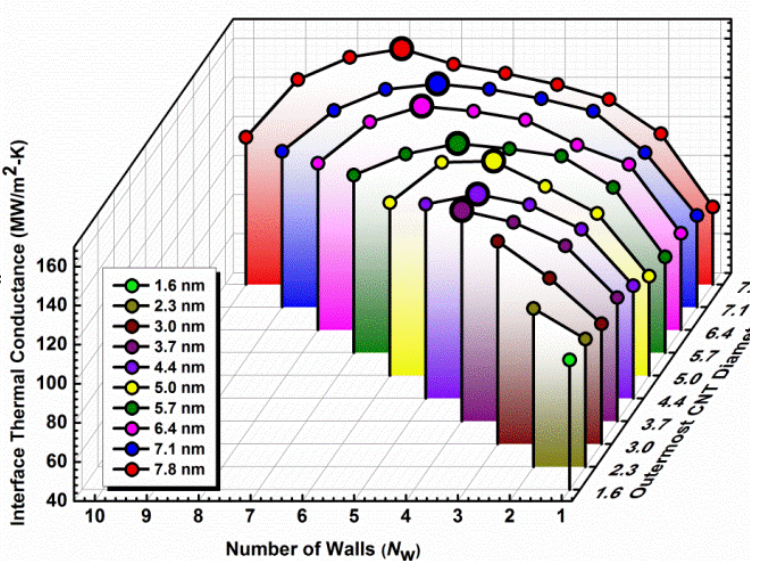

(b)

Figure 4: 3D plot showing interface thermal conductance for different studied MWCNTs as a function of number of walls $\left(N_{\mathrm{W}}\right)$ and outermost CNT diameter (D) for (a) $25 \mathrm{~nm}$ and (b) $50 \mathrm{~nm}$ long MWCNT. The peak positions are highlighted with larger symbols for better clarity in both figures.
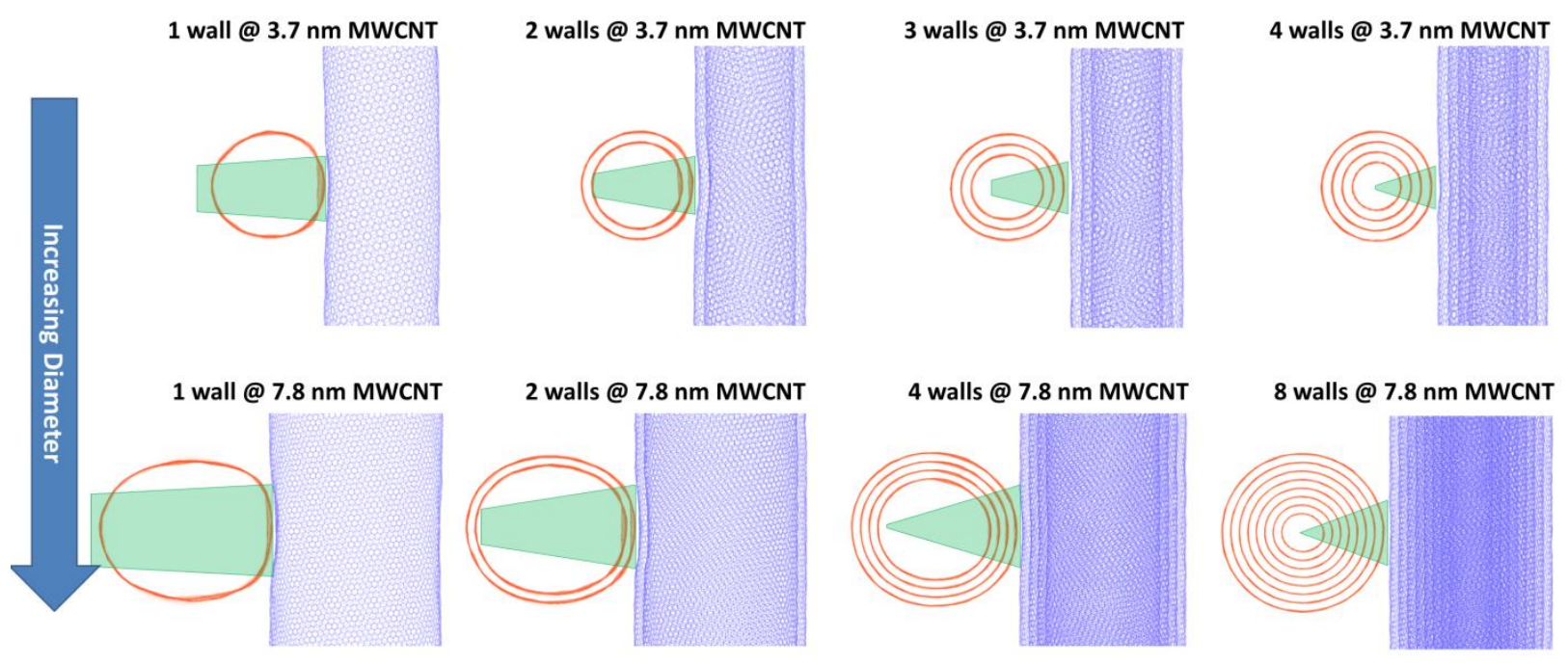

Figure 5: Schematic representations for cross-contact $\left(90^{\circ}\right)$ MWCNT junction for 2 different outer-wall diameters $(D)(3.7 \mathrm{~nm}$ and $7.8 \mathrm{~nm})$ and different number of walls. The figure also schematically depicts the narrowing of the phonon focusing cone (shown in shaded green color) with increase in $N_{\mathrm{W}}$.

Let us first discuss our reasoning behind the observed non-monotonic trend in Figs. 4a and $\mathbf{4 b}$ based on the described premises. Here, the initial increase in conductance with $N_{\mathrm{W}}$ is attributed to the phonon transport characteristics similar to what is observed in flat-graphite and large diameter (> $40 \mathrm{~nm}$ ) MWCNTs [19]. For lower $N_{\mathrm{W}}$, the interacting region is relatively flat (see Fig. 
5), so the radial phonons emitted by hot CNT and get absorbed by cold CNT can propagate radially inward (phonon focusing effect) without much distortion of phonon focusing cone (PFC). Furthermore, they contribute to the thermal conductance if get thermalized in the cold CNT. With increasing $N_{\mathrm{W}}$, greater number of phonons can get thermalized due to more walls (more chance of thermalization before the phonons are reflected from the innermost nanotube), leading to higher values of thermal conductance. On the other hand, large $N_{\mathrm{W}}$ renders the MWCNTs to be significantly stiff and cylindrical, where curvature of the individual CNTs cannot be ignored. The increase in curvature lead to a more focused cone with a larger slope side-wall, leading to less radially propagating phonons aiming at the contact area, as depicted in the Fig. 5. This decrease in 'phonon focusing' region has an adverse effect on thermal conductance, as the region over which they can transport to the other tube significantly reduces, resulting in lowering of thermal conductance with $N_{\mathrm{W}}$. In short, for high and limiting values of $N_{\mathrm{W}}$, the reduced PFC dominates the shown decrease in thermal conductance. Overall, it is the interplay or competition between increased number of walls (thus higher probability of thermalization in the receiving tube and resulting energy transfer) and degree of PFC confining effect, which eventually leads to the observed non-monotonic behavior of thermal conductance as a function of $N_{\mathrm{W}}$.

Now, let us briefly discuss the effect of MWCNT diameter $(D)$ on thermal conductance for constant values of $N_{\mathrm{W}}$ as shown in Fig 4. In such cases, while the thermal conductance only changes marginally for low values of $N_{\mathrm{W}}$, we observe an initial increase followed by eventual saturation of thermal conductance as a function of $D$ for large values of $N_{\mathrm{W}}$. The increasing trend can also be explained using the discussed premises. For small diameter MWCNTs, incorporation of only few walls can sufficiently distort the PFC as shown in Fig. 5, leading to low values of thermal conductance. As the diameter increases, MWCNTs locally adapt their configurations to reduce the local curvature and confinement of the PFC, resulting in the increase of the thermal conductance with increase in diameter as discussed previously. At sufficiently large diameters, the PFC confinement becomes negligible. In such cases, the total phonon scattering probability becomes proportional to wall thickness (constant in this case), resulting in saturation of thermal conductance [19].

Next, we further verify the presented arguments with discussion of peak thermal conductance position (large data points in Fig. 4) with respect to MWCNT diameter and length. For example, 
in Fig. 4a, the peak occurs at $N_{\mathrm{W}}=3$ for $3.7 \mathrm{~nm}$ diameter (large purple rectangle) and moves to $N_{\mathrm{W}}=5$ for $7.8 \mathrm{~nm}$ diameter MWCNT (large red rectangle), which further moves to $N_{\mathrm{W}}=7$ for longer MWCNT (50 nm; large red circle) in Fig. $\mathbf{4 b}$. For small $D$, only a few number of walls can introduce significant curvature in the interacting region, leading to early transition (nonmonotonic behavior) between dominant mechanisms, i.e. from adding more walls to significant altering of the PFC that govern the overall thermal conductance. As MWCNT flexibility increases with increase in $D$, it takes higher number of walls for the transition to happen, resulting in a larger value of peak $N_{\mathrm{W}}$. A similar shift in peak position to larger $N_{\mathrm{W}}$ is also observed with the increase in MWCNT length. As longer CNTs are expected to be more flexible, the CNTs can adapt better for increased interaction near the contact zone with reduced local curvature, and thus, can incorporate more number of inner CNTs which positively contribute towards interface thermal conductance, before confining effects of the PFC become significant.

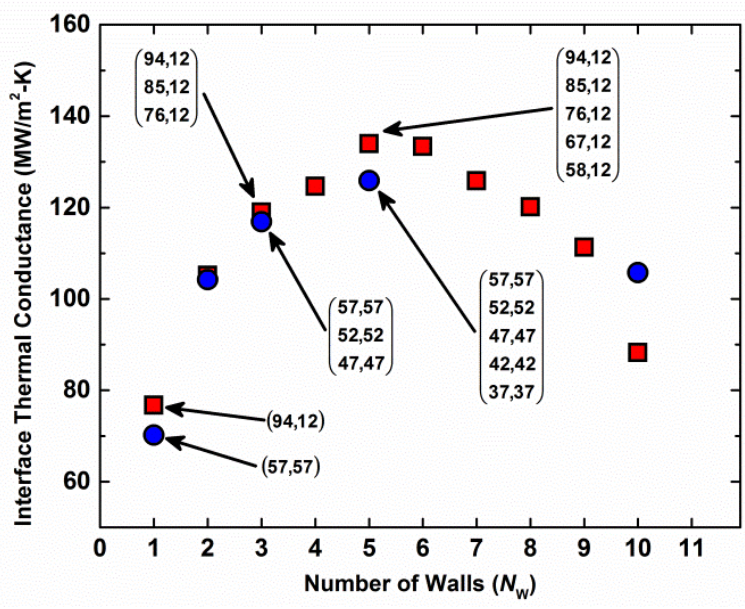

(a)

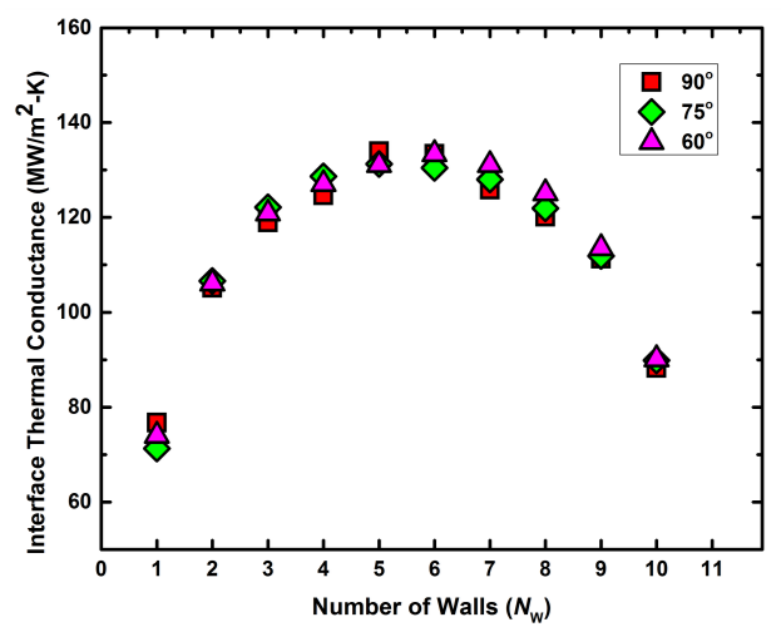

(b)

Figure 6: (a) Plot of interface thermal conductance for two sets of MWCNTs having different inter-layer chirality configurations. Squares represent chiral MWCNTs of type $(n, 12)$ while circles represent arm-chair $(n, n)$ type MWCNTs. For these simulations, the respective outer-wall chirality of achiral and arm-chair MWCNTs was kept constant $((94,12)$ and $(57,57)$ corresponding to $7.8 \mathrm{~nm}$ diameter) while adding inner walls, as represented in abscissa. The chiral characters of both data sets are also shown in the figure for few cases and are tabulated in Table 2 in supporting information S2; (b) Plot of interface thermal conductance vs. number of walls for $8 \mathrm{~nm}$ MWCNT for different studied angular orientations of MWCNTs at the contact. 


\subsection{Effect of Nanotube Chirality and Angular Orientation of the Contacts}

It is well known that there is no specific chiral correlation between consecutive walls in MWCNTs. In order to verify that the observed non-monotonic behavior is not due to chiral angle differences between the successive CNTs, and are in fact associated with the increase in MWCNT curvature irrespective of the chiral angle, we performed another series of contact thermal conductance simulations where all the CNTs within a MWCNT had armchair chirality with the chiral index values ranging from $(12,12)$ to $(57,57)$. The comparison of chiral details is listed in Table 2 in Supporting Information S2. The estimated results for both cases (armchair and achiral MWCNTs) are plotted in Fig. 6a as a function of $N_{\mathrm{W}}$ for $7.8 \mathrm{~nm}$ MWCNTs. On comparing both cases, it is observed that while chirality differences may have some effects at large value of $N_{\mathrm{W}}$ (because of differences in local inter-wall vibrational coupling associated with slight differences in relative diameters of achiral vs arm-chair MWCNTs), the observation of nonmonotonic behavior in both cases, nevertheless, affirms that the presented arguments are indeed associated with the aforementioned proposed premises rather than specific chiral arrangement which somewhat influences the absolute values of contact thermal conductance. For the sake of completeness, we also carried out a set of simulations focusing on how the angular orientation between the MWCNTs modifies the heat flux and the contact thermal conductance. Here, we studied orientation angles of $75^{\circ}$ and $60^{\circ}$ in addition to orthogonal case $\left(90^{\circ}\right)$. An increased total heat flux was observed with decreasing angle because of higher overlap of contact region (Please refer to Supporting Information S4 for the concerned plots), but no differences in the area normalized contact thermal conductance was observed as shown in Fig. $\mathbf{6 b}$.

\section{Conclusions}

Finally, let us put the discussed results in a broader perspective. Our results address and bridge an important, yet previously unreported gap towards the understanding of crucial parameters which govern the thermal conductance across MWCNT contacts. Using phonon focusing and phonon reflection associated with large mean free path, the results demonstrate that the number of walls and associated curvature, both, play a governing role in determining the efficiency of thermal energy exchange across MWCNT contacts, with each of them dominating in different diameter regimes. MWCNT curvature dominates the thermal transport characteristics at small diameters and leads to non-monotonic behavior of contact thermal conductance as a func- 
tion of number of walls. With increasing diameter, however, the effects of curvature start to diminish as inner walls can locally adapt themselves near the interaction zone for optimal thermal transport. In such cases, the thermal transport across the contacts is predominantly governed by number of walls within MWCNTs, as also reported by Yang et al. and can be adequately modeled using simplified flat-graphitic models [19].

From the standpoint of conductive nanoelectronics, where MWCNTs are expected to be utilized as electronic pathways (such as flexible traces, conductive adhesives, nanocomposites), large diameter MWCNTs (with optimal number of CNT walls) are recommended to be exploited as; a) several walls can distribute the electron conduction load; and b) temperature differences at the MWCNT interconnects is expected to be lower for given heat flux due to optimal value of thermal conductance. These recommendations should augment the performance, efficiency lifetime, and thus the reliability of the nanoelectronic devices.

\section{Methods}

\subsection{Simulation Setup: Graphite Nano-ribbons}

A series of MD simulations were performed on flat multi-layer graphene nano-ribbons (MLGNRs) where the number of layers was varied from 1 to 32 (See Fig. 1a). The consecutive layers in graphene nanoribbons (GNRs) were stacked using AB Bernal stacking [3]. The width of the MLGNRs was considered to be $8 \mathrm{~nm}$ (similar to largest diameter MWCNT; see below) while the length was kept to be $25 \mathrm{~nm}$. Although, we realize the experimental unfeasibility of studied MLGNRs, the comparative study provides valuable and comparative insights towards thermal energy exchange occurring across physically interacting carbon nanostructures and also helped us verify results and thermal energy transport mechanisms, as reported by Yang et al.[19] All simulations were performed using LAMMPS molecular dynamics software[41] using PCFF force field [42]. In PCFF force-field, the van der Waals interactions were handled via LenardJones interaction in its 9-6 form as implemented in LAMMPS with interaction cutoff value of 12 $\AA$ Á. This force field has been successfully employed for investigation of thermal conductance/conductivity in several CNTs related studies [35, 43-49]. The further justification and validation of PCFF force-field is further noted in Supporting Information S5. 


\subsection{Simulation Setup: MWCNTs}

Initially, several SWCNTs unit-cells were generated using Materials Studio® with diameter ranging from $1.6 \mathrm{~nm}$ to $8 \mathrm{~nm}$ having different chiral angles $\left(\sim 6^{\circ}-30^{\circ}\right)$. As the unit-cells (repeat unit along axial direction) of the generated chiral SWCNTs have different length, SWCNTs with predefined lengths $(L)$ of $25 \mathrm{~nm}$ and $50 \mathrm{~nm}$ were generated by replicating the unit cell appropriate number of times to a longer length $L^{\prime}\left(L^{\prime}>L\right)$, followed by clipping atoms from the ends to retain desired length $L$. Subsequently, a series of MWCNTs with number of walls $\left(N_{\mathrm{w}}\right)$ varying from 1 to 10 were generated using an in-house script that combined previously clipped several SWCNTs structures to single MWCNT structure. Then, cross-contact physical interconnects of two identical MWCNTs were generated with spacing of $3.4 \AA$ using replication (to create the second MWCNT) followed by mathematical transformations (translation, rotation) using a separate in-house script. A representative MWCNT cross-contact is shown in Fig 1b. The specifics (chirality, diameter, number of walls) of different studied MWCNTs are tabulated in Table 1 in Supporting Information S1. A few other MWCNTs systems were also modeled to investigate effects of differences in inter-wall chirality and angular orientation and are pointed in appropriate section in main text. Non-periodic boundary conditions were used in all 3-directions, where the cross-shaped MWCNT system resides in a relatively larger simulation box.

\subsection{Equilibration}

For equilibration, all simulations were subjected to equilibration at $300 \mathrm{~K}$ using Nose-Hoover thermostating scheme at simulations for 200-500 ps (depending upon system size) where edges ( 1 nm) of the MWCNTs (or MLGNRs) were kept fixed. As the length of the MWCNTs was fixed (because of fixed edges), the initial $\mathrm{C}-\mathrm{C}$ bond lengths were equilibrated to make sure that there was no residual tensile/compressive stress/strain within the CNTs during equilibration. This was verified by monitoring their axial pressure components. ${ }^{\dagger}$ A timestep of $1 \mathrm{fs}$ was used for all simulations during equilibration stage.

\footnotetext{
* For example, unit cell of $(40,12) \mathrm{CNT}$ is $\sim 50.2 \AA$. To create a $250 \AA$ (L) long CNT, the periodic cell was replicated $\sim 6$ times to create $301.2 \AA$ ( (L') CNT. Then, atoms pertaining to $25.6 \AA$ from both ends were removed to create $30 \mathrm{~nm}$ long CNT.

${ }^{\dagger}$ In order to achieve zero axial residual strain for our systems of interest, a test case of cross-contact CNTs was run with different initial C-C bond lengths (between 1.41 and 1.43) at 300K and axial pressure was monitored. Based
} 


\subsection{Thermal Transport Simulations}

After the equilibration, non-equilibrium molecular dynamics (NEMD) simulations were used to calculate thermal conductance at different studied MLGNR and MWCNT interfaces. Similar to equilibrium simulations, the edges $(\sim 1 \mathrm{~nm})$ on both ends of the MLGNRs (and MWCNTs) were kept fixed during the course of NEMD simulations. To introduce temperature drop (or discontinuity) at the interface, $3 \mathrm{~nm}$ sections near both edges (sections of nanotubes in MWCNTs and layers in MLGNRs) were thermostated at $350 \mathrm{~K}$ (hot) and $250 \mathrm{~K}$ (cold) for all studied systems as shown in insets of Fig. 1. All simulations were performed with the timestep of 0.5 fs under NVE (microcanonical) ensemble. To investigate possible artifacts caused by the choice of thermostat, several thermostats were tested (heating/cooling either at the boundaries or across the whole CNTs, using either Nose-Hoover or temperature rescaling thermostats with different updating time intervals). However, negligible differences were observed with no statistically significant trends as also reported previously [50]. For all the results presented further, the temperature rescaling approach was used in the thermostated regions $(3 \mathrm{~nm})$ with rescaling time-interval of 100 timesteps (50 fs). Once the steady state was reached ( $\sim 00 \mathrm{ps})$, the thermal conductance values were calculated using the following equation

$$
\Lambda_{\mathrm{MW} / \mathrm{ML}}=\frac{Q}{A_{\mathrm{MW} / \mathrm{ML}} \Delta T}
$$

where $\Lambda\left(\mathrm{MW} / \mathrm{m}^{2}-\mathrm{K}\right)$ is the area-normalized interface thermal conductance, $Q$ is the heat flow rate, $\Delta T$ is the temperature drop at the interface, and $A_{\mathrm{MW}}$ is the effective area of interaction between cross-contact pair of MWCNTs as discussed in the main text. For MLGNRs, geometric overlapping area was used for $\Lambda$ calculations.

\section{Acknowledgements}

The authors acknowledge US Air Force Office of Scientific Research (AFOSR) for the financial support (Lab Task \# 2306AR8P: PM-Dr Ali Sayir) and Department of Defense Supercomputing Research Center (DSRC) for computational resources to carry out the simulations.

\section{Supporting Information}

on these simulations, the case with zero axial pressure was used as standard length for all studied simulations afterwards. 
Details on specifics of studied MWCNT systems (CNT diameter, number of walls, specific chirality of each CNT, chiral angles, inter-wall chirality differences), pictorial representation of MWCNT near interface region showcasing the different degrees of flattening, thermal conductance data pertaining to different angular orientations of MWCNTs for the studied cases, justification and validation of of PCFF force-field for carrying out this study, as well as sample statistical (and standard error) data for nanotube temperature, employed for thermal conductance calculations. 


\section{References}

[1] C.P. Wong, K.-s. Moon, Nanomaterials for microelectronic and bio-packaging, in: C.P. Wong, K.-S. Moon, Y. Li (Eds.), Nano-bio- electronic, photonic and mems packaging, Springer US, New York, 2010.

[2] R. Saito, G. Dresselhaus, M.S. Dresselhaus, Physical properties of carbon nanotubes, Imperial College Press 1998.

[3] E. Pop, V. Varshney, A.K. Roy, Thermal properties of graphene: Fundamentals and applications, MRS Bull. 37 (12) (2012) 1273-1281.

[4] P. Avouris, F. Xia, Graphene applications in electronics and photonics, MRS Bull. 37 (12) (2012) 1225-1234.

[5] A.M. Marconnet, M.A. Panzer, K.E. Goodson, Thermal conduction phenomena in carbon nanotubes and related nanostructured materials, Rev. Mod. Phys. 85 (3) (2013) 1295-1326.

[6] F.N. Ishikawa, H.-k. Chang, K. Ryu, P.-c. Chen, A. Badmaev, L. Gomez De Arco, et al., Transparent electronics based on transfer printed aligned carbon nanotubes on rigid and flexible substrates, ACS nano 3 (1) (2008) 73-79.

[7] K. Rao, B. Radha, K.C. Smith, T.S. Fisher, G. Kulkarni, Solution-processed soldering of carbon nanotubes for flexible electronics, Nanotechnology 24 (7) (2013) 075301.

[8] A.C. Allen, E. Sunden, A. Cannon, S. Graham, W. King, Nanomaterial transfer using hot embossing for flexible electronic devices, Appl. Phys. Lett. 88 (8) (2006) 083112.

[9] A. Behnam, V.K. Sangwan, X. Zhong, F. Lian, D. Estrada, D. Jariwala, et al., High-field transport and thermal reliability of sorted carbon nanotube network devices, ACS nano 7 (1) (2013) 482-490.

[10] N. Atar, E. Grossman, I. Gouzman, A. Bolker, Y. Hanein, Reinforced carbon nanotubes as electrically conducting and flexible films for space applications, ACS Appl. Mater. Interfaces 6 (22) (2014) 20400-20407.

[11] A. Lekawa-Raus, J. Patmore, L. Kurzepa, J. Bulmer, K. Koziol, Electrical properties of carbon nanotube based fibers and their future use in electrical wiring, Adv. Funct. Mater. 24 (24) (2014) 3661-3682.

[12] Y. Wang, M. Li, Y. Gu, X. Zhang, S. Wang, Q. Li, et al., Tuning carbon nanotube assembly for flexible, strong and conductive films, Nanoscale 7 (7) (2015) 3060-3066.

[13] S. Yao, Y. Zhu, Nanomaterial-enabled stretchable conductors: Strategies, materials and devices, Adv. Mater. 27 (9) (2015) 1480-1511.

[14] P.-C. Ma, N.A. Siddiqui, G. Marom, J.-K. Kim, Dispersion and functionalization of carbon nanotubes for polymer-based nanocomposites: A review, Compos. Part A-Appl. S. 41 (10) (2010) 1345-1367.

[15] R. Prasher, Thermal boundary resistance and thermal conductivity of multiwalled carbon nanotubes, Phys. Rev. B. 77 (7) (2008) 075424.

[16] Y. Yamada, T. Nishiyama, T. Yasuhara, K. Takahashi, Thermal boundary conductance between multi-walled carbon nanotubes, J Therm. Sci. Tech.-Jpn 7 (1) (2012) 190-198.

[17] J. Yang, S. Waltermire, Y. Chen, A.A. Zinn, T.T. Xu, D. Li, Contact thermal resistance between individual multiwall carbon nanotubes, Appl. Phys. Lett. 96 (2) (2010) 023109.

[18] B.A. Cola, J. Xu, C. Cheng, X. Xu, T.S. Fisher, H. Hu, Photoacoustic characterization of carbon nanotube array thermal interfaces, J. Appl. Phys. 101 (5) (2007) 054313.

[19] J. Yang, M. Shen, Y. Yang, W.J. Evans, Z. Wei, W. Chen, et al., Phonon transport through point contacts between graphitic nanomaterials, Phys. Rev. Lett. 112 (20) (2014) 205901.

[20] R.S. Prasher, X.J. Hu, Y. Chalopin, N. Mingo, K. Lofgreen, S. Volz, et al., Turning carbon nanotubes from exceptional heat conductors into insulators, Phys. Rev. Lett. 102 (10) (2009) 105901.

[21] W.J. Evans, M. Shen, P. Keblinski, Inter-tube thermal conductance in carbon nanotubes arrays and bundles: Effects of contact area and pressure, Appl. Phys. Lett. 100 (26) (2012) 261908.

[22] W.J. Evans, P. Keblinski, Thermal conductivity of carbon nanotube cross-bar structures, Nanotechnology 21 (47) (2010) 475704.

[23] H. Zhong, J.R. Lukes, Interfacial thermal resistance between carbon nanotubes: Molecular dynamics simulations and analytical thermal modeling, Phys. Rev. B. 74 (12) (2006) 125403.

[24] S. Maruyama, Y. Igarashi, Y. Taniguchi, J. Shiomi, Anisotropic heat transfer of single-walled carbon nanotubes, J Therm. Sci. Tech.-Jpn 1 (2006) 138-148.

[25] Z. Xu, M.J. Buehler, Nanoengineering heat transfer performance at carbon nanotube interfaces, ACS nano 3 (9) (2009) 2767-2775.

[26] D. Konatham, D.V. Papavassiliou, A. Striolo, Thermal boundary resistance at the graphene-graphene interface estimated by molecular dynamics simulations, Chem. Phys. Lett. 527 (0) (2012) 47-50.

[27] Z. Wei, Z. Ni, K. Bi, M. Chen, Y. Chen, Interfacial thermal resistance in multilayer graphene structures, Phys. Lett. A 375 (8) (2011) 1195-1199. 
[28] V. Varshney, S.S. Patnaik, A.K. Roy, B.L. Farmer, Modeling of thermal conductance at transverse cnt-cnt interfaces, J. Phys. Chem. C 114 (39) (2010) 16223-16228.

[29] K. Bui, B.P. Grady, D.V. Papavassiliou, Heat transfer in high volume fraction cnt nanocomposites: Effects of inter-nanotube thermal resistance, Chem. Phys. Lett. 508 (4-6) (2011) 248-251.

[30] G.-J. Hu, B.-Y. Cao, Thermal resistance between crossed carbon nanotubes: Molecular dynamics simulations and analytical modeling, J. Appl. Phys. 114 (22) (2013) 224308.

[31] A.N. Volkov, R.N. Salaway, L.V. Zhigilei, Atomistic simulations, mesoscopic modeling, and theoretical analysis of thermal conductivity of bundles composed of carbon nanotubes, J. Appl. Phys. 114 (10) (2013) 104301.

[32] M.R. Gharib-Zahedi, M. Tafazzoli, M.C. Bohm, M. Alaghemandi, Transversal thermal transport in singlewalled carbon nanotube bundles: Influence of axial stretching and intertube bonding, J. Chem. Phys. 139 (18) (2013) 184704.

[33] K. Bui, H. Nguyen, C. Cousin, A. Striolo, D.V. Papavassiliou, Thermal behavior of double-walled carbon nanotubes and evidence of thermal rectification, J. Phys. Chem. C 116 (7) (2012) 4449-4454.

[34] L. Hu, A.J.H. McGaughey, Thermal conductance of the junction between single-walled carbon nanotubes, Appl. Phys. Lett. 105 (19) (2014) 193104.

[35] V. Varshney, J. Lee, B.L. Farmer, A.A. Voevodin, A.K. Roy, Modeling of cross-plane interface thermal conductance between graphene nano-ribbons, 2D Mater. 1 (2) (2014) 025005.

[36] A. Liao, R. Alizadegan, Z.-Y. Ong, S. Dutta, F. Xiong, K.J. Hsia, et al., Thermal dissipation and variability in electrical breakdown of carbon nanotube devices, Phys. Rev. B. 82 (20) (2010) 205406.

[37] E. Swartz, R. Pohl, Thermal boundary resistance, Rev. Mod. Phys. 61 (3) (1989) 605-668.

[38] R. Prasher, Predicting the thermal resistance of nanosized constrictions, Nano Lett. 5 (11) (2005) $2155-2159$.

[39] Z. Wei, Y. Chen, C. Dames, Negative correlation between in-plane bonding strength and cross-plane thermal conductivity in a model layered material, Appl. Phys. Lett. 102 (1) (2013) 011901.

[40] J.P. Wolfe, Imaging phonons: Acoustic wave propagation in solids, Cambridge University Press, Cambridge, England, 2005.

[41] S. Plimpton, Fast parallel algorithms for short-range molecular dynamics, J. Comput. Phys. 117 (1) (1995) 119.

[42] H. Sun, S.J. Mumby, J.R. Maple, A.T. Hagler, An ab initio cff93 all-atom force field for polycarbonates, J. Am. Chem. Soc. 116 (7) (1994) 2978-2987.

[43] S. Shenogin, L. Xue, R. Ozisik, P. Keblinski, D.G. Cahill, Role of thermal boundary resistance on the heat flow in carbon-nanotube composites, J. Appl. Phys. 95 (12) (2004) 8136-8144.

[44] M. Hu, P. Keblinski, J.-S. Wang, N. Raravikar, Interfacial thermal conductance between silicon and a vertical carbon nanotube, J. Appl. Phys. 104 (8) (2008) 083503.

[45] T.C. Clancy, T.S. Gates, Modeling of interfacial modification effects on thermal conductivity of carbon nanotube composites, Polymer 47 (16) (2006) 5990-5996.

[46] J. Liu, M. Alhashme, R. Yang, Thermal transport across carbon nanotubes connected by molecular linkers, Carbon 50 (3) (2012) 1063-1070.

[47] V. Varshney, J. Lee, A.K. Roy, B.L. Farmer, Modeling of interface thermal conductance in longitudinally connected carbon nanotube junctions, J. Appl. Phys. 109 (8) (2011) 084913.

[48] J. Lee, V. Varshney, A.K. Roy, B.L. Farmer, Single mode phonon energy transmission in functionalized carbon nanotubes, J. Chem. Phys. 135 (10) (2011) 104109.

[49] S.T. Huxtable, D.G. Cahill, S. Shenogin, L. Xue, R. Ozisik, P. Barone, et al., Interfacial heat flow in carbon nanotube suspensions, Nat. Mater. 2 (11) (2003) 731-734.

[50] V. Varshney, A.K. Roy, D.S. Dudis, J. Lee, B.L. Farmer, A novel nano-configuration for thermoelectrics: Helicity induced thermal conductivity reduction in nanowires, Nanoscale 4 (16) (2012) 5009-5016. 
